\title{
BMJ Open Effectiveness and cost-effectiveness of a combined lifestyle intervention compared with usual care for patients with early-stage knee osteoarthritis who are overweight (LITE): protocol for a randomised controlled trial
}

\author{
Nuria E J Jansen (10 , , Dieuwke Schiphof, ${ }^{1}$ Edwin Oei, ${ }^{2}$ Judith Bosmans, ${ }^{3}$ \\ Jolande van Teeffelen, ${ }^{4}$ Anita Feleus, ${ }^{5}$ Jos Runhaar, ${ }^{1}$ Joyce van Meurs, ${ }^{6}$ \\ Sita M A Bierma-Zeinstra, ${ }^{1,7}$ Marienke van Middelkoop ${ }^{1}$
}

To cite: Jansen NEJ, Schiphof D, 0ei E, et al. Effectiveness and costeffectiveness of a combined lifestyle intervention compared with usual care for patients with early-stage knee osteoarthritis who are overweight (LITE) protocol for a randomised controlled trial. BMJ Open 2022;12:e059554. doi:10.1136/ bmjopen-2021-059554

- Prepublication history and additional supplemental material for this paper are available online. To view these files, please visit the journal online (http://dx.doi.org/10.1136/ bmjopen-2021-059554).

Received 29 November 2021 Accepted 09 February 2022

Check for updates

(c) Author(s) (or their employer(s)) 2022. Re-use permitted under CC BY-NC. No commercial re-use. See rights and permissions. Published by BMJ.

For numbered affiliations see end of article.

Correspondence to

Nuria E J Jansen;

n.jansen@erasmusmc.nl

\section{ABSTRACT}

Introduction Obesity is the most important modifiable risk factor for knee osteoarthritis (KOA). Especially in an early stage of the disease, weight loss is important to prevent further clinical and structural progression. Since 2019, general practitioners (GPs) in the Netherlands can refer eligible patients to a combined lifestyle intervention (GLI) to promote physical activity, healthy nutrition and behavioural change. However, GPs scarcely refer patients with KOA to the GLI potentially due to a lack of evidence about the (cost-)effectiveness. The aim of this study is to determine the (cost-)effectiveness of the GLI for patients with early-stage KOA in primary care.

Methods and analysis For this pragmatic, multi-centre randomised controlled trial, 234 participants (aged 45-70 years) with National Institute for Health and Care Excellence (NICE) guideline diagnosis of clinical KOA and a body mass index above $25 \mathrm{~kg} / \mathrm{m}^{2}$ will be recruited using a range of online and offline strategies and from general practices in the Netherlands. Participants will receive nine 3-monthly questionnaires. In addition, participants will be invited for a physical examination, MRI assessment and blood collection at baseline and at 24-month followup. After the baseline assessment, participants are randomised to receive either the 24-month GLI programme in addition to usual care or usual care only. Primary outcomes are self-reported knee pain over 24 months, structural progression on MRI at 24 months, weight loss at 24 months, as well as societal costs and Quality-Adjusted Life-Years over 24-month follow-up. Analyses will be performed following the intention-to-treat principle using linear mixed-effects regression models.

Ethics and dissemination Ethical approval was obtained through the Medical Ethical Committee of the Erasmus MC University Medical Center Rotterdam, The Netherlands (MEC-2020-0943). All participants will provide written informed consent. The results will be disseminated through publications in peer-reviewed journals, presentations at international conferences and among study participants and healthcare professionals.
Strengths and limitations of this study

The trial is pragmatic in design and will be performed in multiple centres, enabling better generalisability.

- The combined lifestyle intervention (GLI) is individualised based on the patient's personal goals but also relies on the input of the healthcare professionals involved in the GLI, which may introduce clinical heterogeneity.

- Further follow-up is required to assess long-term benefits after the end of the intervention.

- A mixed-methods approach using quantitative and qualitative methods will support future implementation.

Trial registration number Netherlands Trial Registry (NL9355).

\section{INTRODUCTION}

In almost all the countries, the prevalence of individuals who are overweight is rising. ${ }^{1}$ Having a body mass index (BMI) greater than 25 increases the risk of many chronic diseases, including cardiovascular disease, diabetes mellitus (DM), chronic kidney disease, multiple cancers, depression and musculoskeletal disorders such as osteoarthritis (OA). ${ }^{2} \mathrm{OA}$ is a joint disorder, most frequently affecting the knee, hand, hip and foot joints. ${ }^{3}$ The prevalence of $\mathrm{OA}$ in the Netherlands is expected to increase from $7 \%$ in 2015 to $12 \%$ in 2040 , which is mainly attributed to rising overweight rates. ${ }^{4}$ Patients with knee OA (KOA) experience pain and impaired physical functioning, which may affect their quality of life. KOA is associated with many 
risk factors, but most strongly with older age, female sex, high BMI, previous knee injury, knee malalignment and genetic predisposition..$^{5-8}$ Although a high BMI is considered the most modifiable risk factor, management of KOA is mainly restricted to diminishing pain and disability with pain medication and exercise therapy. ${ }^{9}$ If conservative management is unsuccessful, total knee arthroplasty (TKA) is the last treatment option for patients with advanced KOA suffering from severe pain. ${ }^{10}$ Due to the progressive nature of the disease and the absence of a disease-modifying osteoarthritis drug, the average costs for a patient with KOA remain high throughout the course of the disease. ${ }^{11}$ In 2017, the medical costs of KOA have been estimated at 488 million Euros, accounting for $1.4 \%$ of all healthcare costs in the Netherlands. ${ }^{12}$

Several trials showed that body weight loss has a positive effect on knee pain and function ${ }^{13-16}$ and was found to reduce the risk for the onset of radiographic KOA. ${ }^{17}$ Therefore, guidelines recommend $5 \%-10 \%$ wt loss for patients with KOA who are overweight. ${ }^{18}$ So far, all studies assessing the effects of weight-loss have been performed in patients with established KOA. Although studies have recognised the importance of early identification and treatment of KOA, ${ }^{19} 20$ the effectiveness of weight loss in patients with early-stage KOA is unknown up until now. Early intervention increases the chance to prevent or slow progression towards end-stage KOA, when a costly TKA is required.

Since 2019, general practitioners (GPs) in the Netherlands can refer eligible patients to a combined lifestyle intervention (GLI). The general aim of the GLI is to lose at least $5 \%$ of body weight by promoting physical activity (PA), healthy nutrition and behavioural change. The GLI is available for patients who are overweight and with DM type 2, cardiovascular disease, sleep apnoea or OA. GPs, as the first point of contact, play an essential role in early case finding and diagnosis, which enables GPs to intervene in an early stage of KOA. Although GPs can refer patients with KOA to the GLI, it is not frequently done in routine clinical practice, potentially due to a lack of evidence about the clinical effectiveness. A longitudinal study showed that the GLI leads to a positive change in behavioural lifestyle factors and a reduction in body weight in patients who are obese. ${ }^{21}$ However, it is unclear whether the GLI is effective for patients with early-stage KOA. There are several barriers to weight loss that may pose a challenge for both healthcare professionals (HCPs) and patients, including a desire for high-calorie or high-fat food, ${ }^{22}$ low self-efficacy, ${ }^{23}$ fear of movement, ${ }^{24}$ pain experienced during activities and decreased physical functioning. ${ }^{25}$ To date, there have been no studies examining the (cost-) effectiveness of the GLI in the early-stage KOA population.

\section{Objectives}

The primary objective of this study is to evaluate the effectiveness of the GLI added to usual care in a primary care setting for patients with early-stage KOA who are overweight compared with usual care alone on (1) selfreported knee pain over 24 months, (2) structural OA progression on MRI over 24 months, (3) weight loss at 24 months and for the cost-effectiveness from a societal perspective (4) Quality-Adjusted Life-Years (QALYs) over 24 months.

Secondary objectives are to determine the effect of the GLI on (1) performance-based physical functioning (Timed Up and Go, quadriceps strength) at 24 months, (2) other disease-specific Patient Reported Outcome Measures (PROMs) over 24 months, (3) general health and inflammation-related outcomes at 24 months including blood pressure, waist circumference, biomarkers (glycosylated haemoglobin (HbAlc), total cholesterol, triglycerides, $\mathrm{C}$ reactive protein (CRP), interleukin 6 (IL-6), creatinine) and microbiome composition.

\section{METHODS AND ANALYSIS}

The Standard Protocol Items: Recommendations for Interventional Trials reporting guidelines were used in the development of this study protocol (online supplemental additional file 1$){ }^{26}$

\section{Trial design and setting}

The Lifestyle Intervention Trial for Early-stage KOA (LITE) study is a pragmatic, multi-centre, two-arm, superiority randomised controlled trial (RCT). Patients with early-stage KOA who are overweight (ie, BMI $\geq 25 \mathrm{~kg} / \mathrm{m}^{2}$ ) will be equally randomised to (1) the GLI in addition to usual care or (2) usual care only (figure 1). Multiple GLI programmes are available in the Netherlands ('Slimmer', 'Cool', 'Samen Sportief in Beweging', 'X-Fittt GLI'). ${ }^{27}$ For the current trial, the 'Beweegkuur-gecombineerde leefstijlinterventie' was chosen as the intervention programme. ${ }^{28}$ This decision was based on the greater accessibility of the intervention in the region of Rotterdam and because we expect it to be the best fit for this target population (ie, patients with early-stage KOA who are overweight). Recruitment for the trial began in July 2021 and the aim is to include the last participant by July 2022 . With a 2-year follow-up for all participants, the trial is anticipated to be completed by June 2024 .

\section{Participants}

Two recruitment strategies will be used. Participating GPs can invite eligible patients to participate in the trial. Simultaneously, participants will be recruited with offline and online recruitment strategies (eg, advertisements in newspapers or on social media). Potential participants will be screened by the researchers using the inclusion and exclusion criteria listed in box 1 . Eligible participants will be provided with an information package and consent form (online supplemental additional file 2) and will have the opportunity to ask questions. Researchers will obtain written consent from participants willing to participate in the trial before data collection. GPs of participants who signed up via the online and offline 


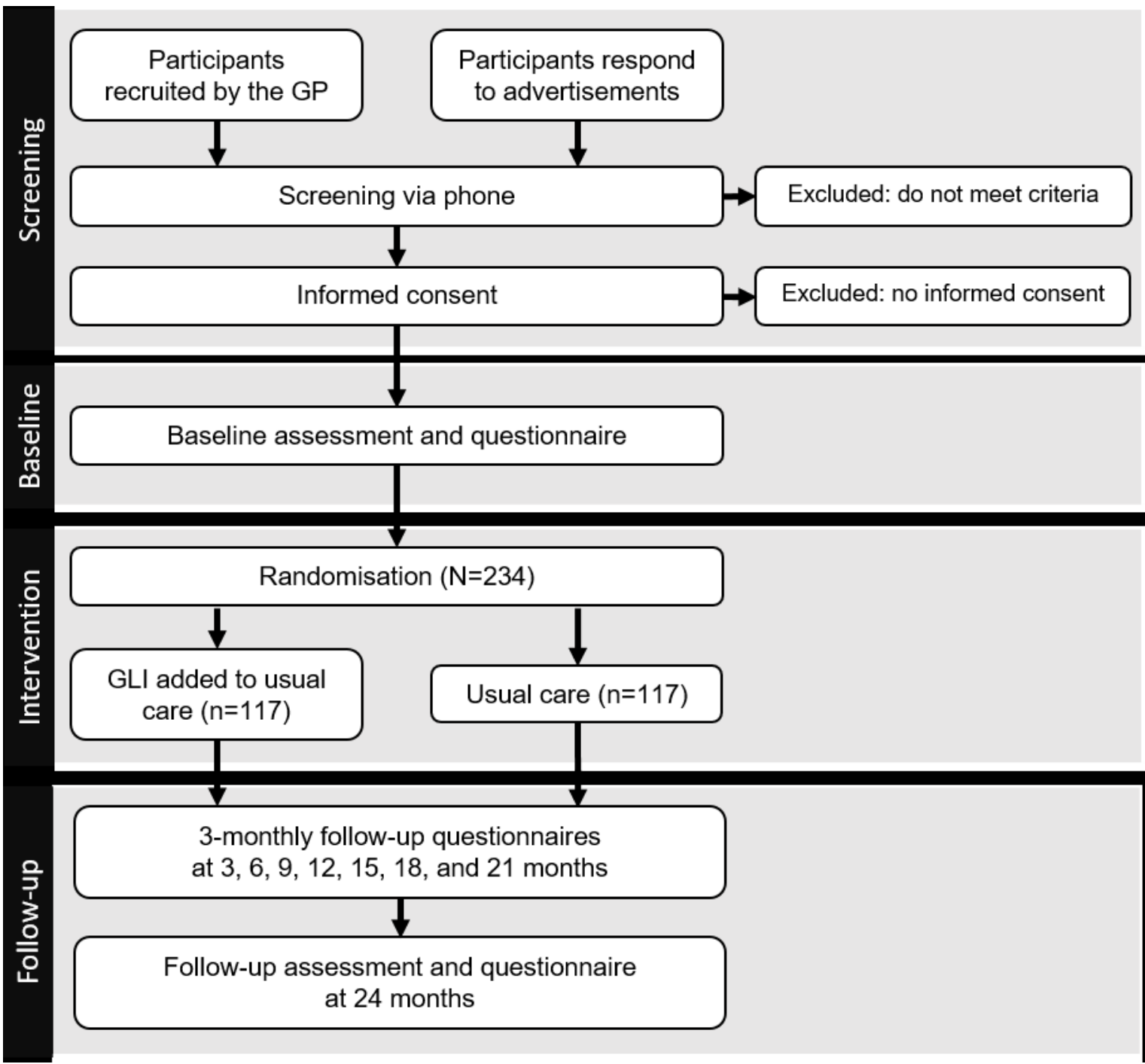

Figure 1 Schematic design of the Lifestyle Intervention Trial for Early-stage KOA trial. GLI, combined lifestyle intervention; GP, general practitioner; KOA, knee osteoarthritis.

recruitment strategies will be informed of trial participation. During the screening, participants will be asked about their most symptomatic knee, which will be considered the index knee for the duration of the trial. If both knees are equally affected, the right knee will be chosen as the index knee.

\section{Allocation and blinding}

After the baseline assessment, participants are randomised with an allocation ratio of $1: 1$ to the intervention or control group. Block randomisation (random size 4 or 6 ) stratified by BMI $\left(\mathrm{BMI} \geq 25 \mathrm{~kg} / \mathrm{m}^{2} / \mathrm{BMI} \geq 30 \mathrm{~kg} / \mathrm{m}^{2}\right)$ will be employed using the electronic data capture (EDC) system Castor (Ciwit BV, Amsterdam, The Netherlands). Blinding of participants, researchers and HCPs is not possible due to the nature of the intervention. Researchers will inform participants and their GPs of their treatment allocation and will inform the GP about participants who have been allocated to the intervention group to request a referral to the GLI.

\section{Interventions}

Combined lifestyle intervention

Participants allocated to the intervention group will receive the GLI in addition to usual care. The 24-month GLI is a multicomponent intervention addressing PA, nutrition and behavioural skills. The content of the GLI is not designed specifically for the target population. The participants will follow the original programme that is individualised based on the participant's personal goals. The general aim of the GLI is to lose at least $5 \%$ of body weight during the first year and to maintain this weight loss in the second year. Participants will be supported by a team of accredited HCPs, including the lifestyle coach (LSC), a physical therapist (PT) and a dietician. The programme consists of individual and group sessions guided by the HCPs (table 1).

\section{Lifestyle coaching}

The LSC is trained in motivational interviewing, a method for autonomy-supportive coaching, which is an essential 
Box 1 Inclusion and exclusion criteria in the Lifestyle Intervention Trial for Early-stage KOA trial

\section{Inclusion criteria}

1. First presentation at the GP with knee complaints within the previous 24 months.

2. Aged $\geq 45$ and $<70$ years.

3. NICE guideline diagnosis of clinical KOA.

- Aged 45 or over.

- Activity-related joint pain.

- Either no morning joint-related stiffness or morning stiffness that lasts no longer than $30 \mathrm{~min}$ min.

4. Presence of overweight $\left(B M I^{*} \geq 25 \mathrm{~kg} / \mathrm{m}^{2}\right)$.

5. Able to give written informed consent.

\section{Exclusion criteria}

1. Other pathological conditions that could explain the joint complaints, including traumatic onset knee complaints or the presence of other forms of arthritis (eg, rheumatoid arthritis, psoriatic arthritis), prepatellar bursitis or patellar tendinitis.

2. Any lower extremity condition other than KOA resulting in physical impairment that will limit GLI participation.

3. Contraindications for MRI

4. Previously participated in a GLI.

5. Not being able to speak, read or write Dutch.

BMI, body mass index; GLI, combined lifestyle intervention; GP, general practice; KOA, knee osteoarthritis; NICE, National Institute for Health and Care Excellence.

${ }^{*} \mathrm{BMI}$ is calculated as body weight in kilograms divided by the squared height in metres.

part of the GLI. ${ }^{29}{ }^{30}$ The participant is supported by the LSC to become intrinsically motivated to change their behaviour concerning PA and healthy dietary behaviour. The intake with the LSC is aimed at setting personal goals and identifying barriers and facilitators to a healthy lifestyle. Following the intake, the participant will have five individual sessions with the LSC to evaluate their behavioural change.

\section{Exercise component}

The exercise component aims to reach a minimum of $1200 \mathrm{kcal} /$ week of energy expended by PA. The participants will then gradually increase their energy expenditure to $2000 \mathrm{kcal} /$ week by adjusting the frequency, duration and/or intensity of the PA. During the intake with the PT, an exercise programme is formed based on personal goals and the participant's ability to exercise. The PT will support the participant to overcome barriers to exercise and enable the participant to exercise in local facilities. After two sessions with the PT, the participant is introduced to a sports coach to continue with the exercise programme in year 2 .

\section{Nutritional component}

The sessions with the dietician are aimed to improve the nutritional knowledge and skills to structurally adapt the participant's dietary habits. The nutritional advice is based on evidence-based dietary guidelines. ${ }^{31-33}$ A nutritional plan will be formulated during the intake with the dietician. After the intake, the dietician will plan two individual sessions to monitor the participant's eating behaviour and seven group sessions to provide nutritional recommendations, tips to cope with high-risk situations, strategies to prevent relapse and to facilitate interaction between participants.

\section{Usual care}

All participants will receive usual care by their GP following the Nederlands Huisartsen Genootschap guideline for non-traumatic knee complaints. ${ }^{34}$ This includes patient education, promoting weight loss and advising regular PA ( $\geq 30 \mathrm{~min} /$ day), prescribing analgesics or intraarticular corticosteroid injections, or referring patients for specialist treatment (eg, exercise therapy).

\section{Use of co-interventions}

Any care that participants receive concerning weight loss, $\mathrm{PA}$ and use of cointerventions (eg, non-steroidal antiinflammatory drugs, other analgesic drugs, intra-articular injections, knee braces or inlays) will be documented through self-report. Referral for joint replacement or bariatric surgery is allowed. Participants allocated to the control group cannot be referred to the GLI.

\section{Data collection}

Participants will be assessed at nine-time points in the 24-month study period. After providing consent, participants are asked to complete the baseline (T0) questionnaire, prior to the baseline appointment. Thereafter, participants will receive eight 3-monthly (T3-T24) follow-up questionnaires. Questionnaires are sent digitally by Castor EDC and reminders will automatically be sent. The content of the questionnaire per time-point is shown in table 2. Participants will be invited for a clinical examination, 1 hour each, at baseline (T0) and at 24-month follow-up (T24). The examination will be performed by two trained researchers. Adherence to the GLI will be recorded by the HCPs.

\section{Clinical visit}

Physical examination

Body weight $(\mathrm{kg})$, height $(\mathrm{m})$, BMI (calculated) and waist circumference $(\mathrm{mm})$ at the midpoint between the iliac crest and the lowest rib will be measured using standardised techniques. Brachial blood pressure will be measured two times in a seated position on the left arm with 3 min rest between consecutive measurements with an oscillometric device (Omron HBP-1320, Omron Health Care Inc., Illinois, USA). Systolic and diastolic blood pressure is automatically calculated.

Knee function will be examined by assessing joint line tenderness, palpable warmth, ${ }^{35}$ crepitus with active loaded movement of the joint, bony enlargement, painful and restricted passive range of motion measured by goniometry of knee extension and flexion with the participant in a supine position, effusion using the hydrops test $^{36}$ and knee joint alignment (hyperextension, varus or 
Table 1 Combined lifestyle intervention programme with the individual and group sessions per healthcare professional

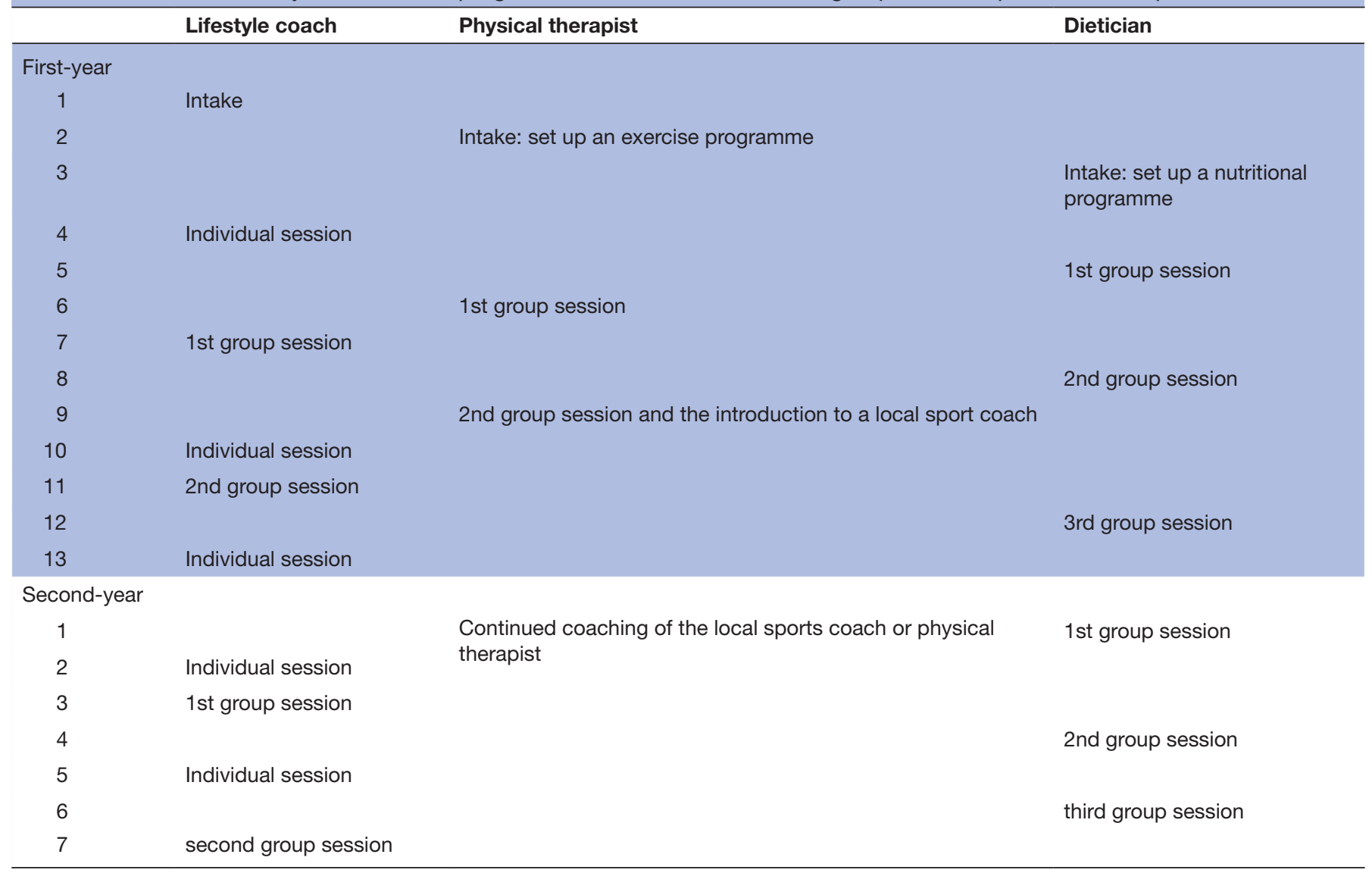

valgus). ${ }^{37}$ The hands will be examined for signs of hand OA (Heberden's and Bouchard's nodes).

Physical function will be assessed with the Timed Up and Go (TUG) and by measuring quadriceps strength. The TUG measures the time (s) needed by participants to stand up from a chair, walk $3 \mathrm{~m}$ as quickly and safely as possible, walk around a cone, return and sit down in the chair. ${ }^{38}$ Participants are allowed to have one practice trial, followed by an actual test.

Maximal voluntary isometric contraction in knee extension (Newton) will be measured with a hand-held dynamometer (HHD) (MicroFET2, Hoggan Health Industries Inc., West Jordan, Utah, USA). Participants will be seated at the examination table with their lower limbs bent over the edge and their arms crossed in front of the body. Participants are instructed to maintain the trunk in the upright position. The HHD will be fixed to the table leg using a fixation belt to allow a maximum of $60^{\circ}$ of knee flexion during the muscle contraction. The test will be performed two times per leg, starting with the side of the index knee, with a rest period of $60 \mathrm{~s}$ between measurements. The HHD will be placed on the anterior and proximal aspect of the tibia at the level of the malleoli. The fixation belt will counter all resistance to knee extension but the researcher will stabilise the HHD to prevent movement. Participants are verbally encouraged to exert maximum muscle power for $3 \mathrm{~s}$.
Mechanical sensitivity to pain will be assessed with the Pressure Pain Threshold (PPT) test using an HHD with a probe size of $1 \mathrm{~cm}^{2}$. Two recordings will be taken at three test sites with the participants lying in a supine position, including the (1) medial joint line of the index knee, (2) medial joint line of the contralateral knee and (3) at the mid-point between the wrist and elbow of the volar aspect of the forearm contralateral to the index knee. Pressure was applied at a constant rate of $5 \mathrm{kPa} / \mathrm{s}$ until the participants felt pain or $70 \mathrm{kPA}$ is reached. The participants will be instructed to say 'stop' when the pressure sensation changes to the first sensation of pain and asked to rate the intensity of pain on an 11-point Numeric Rating Scale (NRS) with 0 representing 'no pain' and 10 representing 'worst pain possible'. The pressure (Newton) corresponding to the PPT will be recorded.

\section{Blood sampling}

Non-fasting venous blood samples will be collected. Serum or plasma concentrations of HbA1c, total cholesterol, triglycerides, CRP, IL-6 and creatinine will be determined by using a standard protocol. The remaining plasma samples will be aliquoted and stored at $-80^{\circ} \mathrm{C}$ for future analyses of relevant biomarkers. Plasma samples will be used to assess targeted proteomics using Olinktechnology (Olink Biosciences, Uppsala, Sweden). ${ }^{39}$ Cell pellets will be kept frozen at $-20^{\circ} \mathrm{C}$ and DNA will be 


\begin{tabular}{|c|c|c|c|c|}
\hline Domain & Measure & Content & Ref & Time \\
\hline \multicolumn{5}{|l|}{ Descriptive variables } \\
\hline \multirow[t]{2}{*}{ Demographics } & & Body weight and height. & & All \\
\hline & & Sex, menopausal status, education level and ethnicity. & & TO \\
\hline \multirow[t]{5}{*}{ Lifestyle and nutrition } & $\begin{array}{l}\text { Short QUestionnaire to ASsess Health- } \\
\text { enhancing physical activity (SQUASH) }\end{array}$ & $\begin{array}{l}\text { The SQUASH assesses perceived PA during a normal } \\
\text { week over the past few months over five domains, } \\
\text { including activities at work, commuting, household } \\
\text { activities, leisure time and sports activities. }\end{array}$ & 48 & TO \\
\hline & $\begin{array}{l}\text { Factor Occupational Rating System } \\
\text { Scale (FORSS) }\end{array}$ & The FORSS assesses PA during work and leisure. & 49 & TO \\
\hline & Sport participation & Type, duration and intensity of past and current sports. & & TO \\
\hline & & Smoking and alcohol consumption. & & T0, T24 \\
\hline & 3-day food diary & $\begin{array}{l}\text { Dietary intake will be assessed with a } 24 \text {-hour food diary } \\
\text { filled in for three consecutive days ( } 2 \text { weekdays and } \\
1 \text { weekend day). }\end{array}$ & & $\begin{array}{l}\text { T0, T6, T12, } \\
\text { T18, T24 }\end{array}$ \\
\hline \multirow[t]{2}{*}{ Knee complaints } & History of knee complaints & $\begin{array}{l}\text { Duration of symptoms, specific patellofemoral pain } \\
\text { features, previous knee injuries (type, year of injury, } \\
\text { onset and treatment) and medication use related to knee } \\
\text { complaints. }\end{array}$ & & TO \\
\hline & Knee complaints & $\begin{array}{l}\text { Flares, the feeling of a swollen knee and the duration of } \\
\text { morning stiffness. }\end{array}$ & & All \\
\hline Comorbidities & $\begin{array}{l}\text { Self-Administered Comorbidity } \\
\text { Questionnaire (SCQ) }\end{array}$ & $\begin{array}{l}\text { The SCQ addresses } 10 \text { medical conditions and three } \\
\text { not prespecified problems, regarding the occurrence } \\
\text { of the problem, received treatment or medication and } \\
\text { experienced limitations in everyday life activities. }\end{array}$ & 50 & TO \\
\hline
\end{tabular}

Primary outcomes

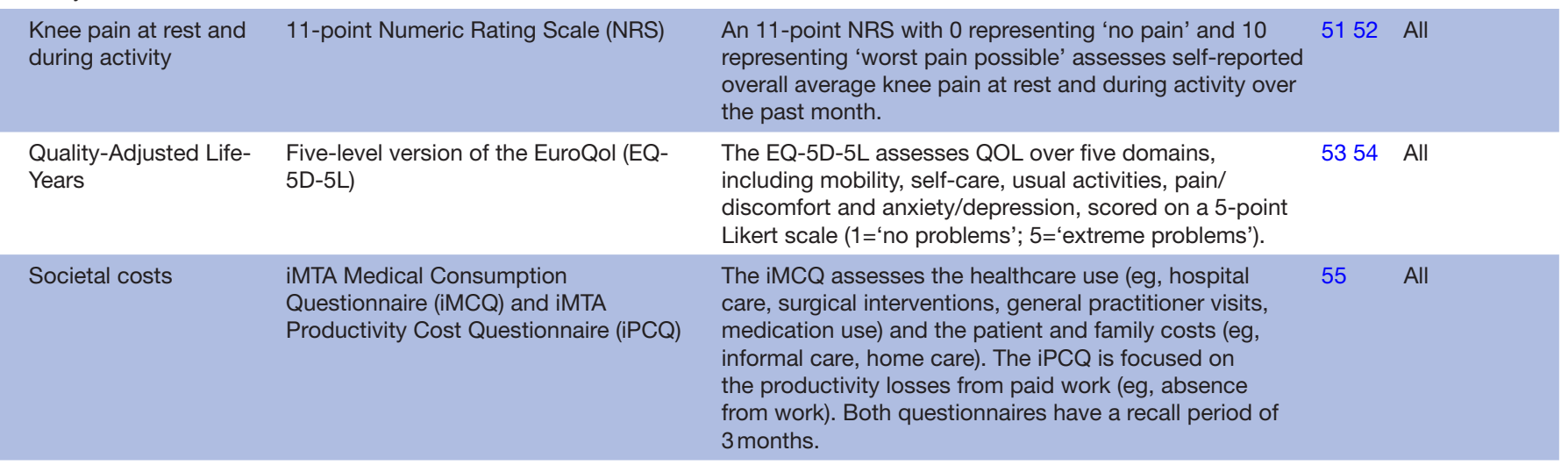

Secondary outcomes

$\begin{array}{ll}\text { Knee complaints } & \text { Knee injury and Osteoarthritis Outcome } \\ \text { Score (KOOS) }\end{array}$
Score (KOOS)

Intermittent and Constant OsteoArthritis Pain (ICOAP)

Global Rating of Change (GRoC)

Patient Acceptable Symptom State (PASS)

\section{The KOOS assesses symptoms and functional} limitations. It consists of five subscales that are scored on a 5-point Likert scale on pain, other symptoms, ADL, sport and recreation, and QOL in the previous 7 days. All the subscales will be included separately.

The ICOAP is comprised 5 -items assessing constant knee pain and 6-items assessing intermittent knee pain in the previous week scored on a 6-point Likert scale.

The GRoC assesses the overall perceived change in KOA 59 complaints on a 7-point Likert scale ranging from 'much worse' to 'much better'.

The PASS assesses whether the current state (eg, consequences of KOA in the past week) would be acceptable or unacceptable if that remained during the rest of their life. 


\begin{tabular}{clll}
\hline Table 2 Continued & & & \\
\hline Domain & Measure & Content & Ref \\
\hline Psychosocial factors & Tampa Scale of Kinesiophobia (TSK) & $\begin{array}{l}\text { The TSK assesses fear of (re-)injury due to movement } \\
\text { with 17-items that is scored on a 4-point Likert scale } \\
\text { ranging from 'strongly agree' to 'strongly disagree'. }\end{array}$ & T0, T24 \\
& Self-Efficacy for Exercise (SEE) & $\begin{array}{l}\text { The SEE assesses the confidence in performing PA three } \\
\text { times a week for 20 min under specified circumstances } \\
\text { (eg, lack of time, experience pain during activity, } \\
\text { experience stress). }\end{array}$ & T0, T12, T24
\end{tabular}

KOA, knee osteoarthritis; PA, physical activity; QOL, quality of life.

isolated. Genetic profiles will be determined using DNAvariant microarray analysis.

\section{Microbiome}

Faecal samples will be collected at baseline and 24-month follow-up and stored frozen. The intestinal microbiome composition will be assessed using 16S-sequencing. ${ }^{40}$ Previously identified microbiome composition that was found to be linked to joint pain and inflammation will be analysed. $^{40}$

\section{MRI}

MRI of the index knee will be performed on a 1.5 Tesla MR unit (MAGNETOM Sola, Siemens Medical Solutions, Erlangen, Germany) with a dedicated 18-channel knee coil. The protocol comprises a sagittal, axial and coronal fast spin-echo proton density-weighted sequence with fat suppression, a sagittal and axial proton densityweighted Dixon sequence with fat suppression, as well as a fast spin-echo T2-weighted water excitation 3D sequence. A screening form will be completed by all participants to ensure there is no contraindication for MRI. Semi-quantitative scoring will be conducted using the MRI Osteoarthritis Knee Score (MOAKS) to evaluate multi-feature joint changes. ${ }^{41}$ The MOAKS evaluates the presence of articular cartilage loss in conjunction with surrounding bony and soft tissue abnormalities, including bone marrow lesions, osteophytes, lesions of the menisci, ligaments and tendons, joint effusion and synovitis, as well as periarticular features. The change of individual features per subregion ( $1=$ 'progression'; $-1=$ 'improvement'; $0=$ 'no change') and the summed change per feature will be assessed. ${ }^{42}$ The summed change scores per feature will be dichotomised into progression versus no progression (change score $\geq 1=$ 'progression'; change score $<1=$ "'no progression').

\section{Outcome measures}

Primary outcome measures

The study will include several primary outcomes.

- Clinical outcome: change in average knee pain at rest and during activity (NRS) over 24 months.

- Structural outcome: structural progression of KOA (MOAKS) on MRI at 24 months.

- Mechanistic outcome: change in body weight at 24 months.

For the cost-effectiveness:
- Societal costs (iMTA Medical Consumption Questionnaire, iMTA Productivity Cost Questionnaire) over 24 months.

- QALYs based on quality of life (Five-level version of the EuroQol (EQ-5D-5L)) over 24 months.

\section{Secondary outcome measures}

- Change in physical functioning (TUG, quadriceps strength) at 24 months.

- Change in disease-specific PROMs (Knee injury and Osteoarthritis Outcome Score, Intermittent and Constant OsteoArthritis Pain, Global Rating of Change, Patient Acceptable Symptom State) over 24 months.

- Change in general health and inflammation-related outcomes (blood pressure, waist circumference, biomarkers (HbAlc, total cholesterol, triglycerides, CRP, IL-6, creatinine) and microbiome composition) at 24 months.

Other variables of interest:

- General and OA-specific characteristics will be collected at baseline including age, sex, menopausal status, employment status, educational level, ethnicity, knee complaints, history of knee injuries, comorbidities (Self-Administered Comorbidity Questionnaire), plasma biomarkers (Olink proteomics) and genetic profiles.

- Lifestyle data will be collected at baseline including PA level (Short QUestionnaire to ASsess Healthenhancing physical activity), past and current sports participation, PA during work and leisure (Factor Occupational Rating System Scale), and smoking and alcohol consumption.

- Change in psychosocial factors (Tampa Scale of Kinesiophobia, Self- Efficacy for Exercise) at 24 months.

- Change in self-reported body weight over 24 months.

- Change in self-reported diet over 24 months.

- Change in knee function at 24 months.

- Change in pain sensitisation (PPT) at 24 months.

\section{Qualitative interviews}

Following the 2-year intervention, semi-structured interviews will be conducted to receive feedback on perceived barriers and facilitators of both participants and HCPs from the GLI. It is anticipated that approximately 15 participants and 10 HCPs will be needed to reach data 
saturation, though data collection will continue until data saturation is reached. The interviews will be conducted by a member of the research team and the interviews will be audiotaped and transcribed verbatim. The topic guides for participants and HCPs will be developed from existing literature based on the study aims of measuring barriers and facilitators for the implementation of the GLI. Transcripts will be analysed using thematic analysis. The primary outcome will be to identify barriers and facilitators of patients and HCPs for the implementation of the GLI for patients with KOA.

\section{Sample size}

A $5 \mathrm{~kg}$ or $5 \% \mathrm{wt}$ loss was used as the intermediate primary outcome to estimate the sample size. In our previously conducted trial, $17 \%$ of the participants in the intervention group who received a diet and exercise programme fulfilled this criterion. ${ }^{17}$ For the sample size calculation of the LITE trial, a similar fulfilment for the intervention group was assumed. It was estimated that $5 \%$ of the participants in the control group will fulfil this criterion. Based on a power of $80 \%$, an alpha of $5 \%$ and a dropout rate of 10\% (loss-to-follow-up and missing data) a sample size of 117 participants in each treatment arm is needed. The sample size also provides at least $95 \%$ power to detect a minimal clinically important difference of 2-points in mean pain (11-point NRS) in our primary symptomatic outcome. $^{43}$

\section{Statistical analysis}

The analysis will be performed using an intention-totreat approach. Descriptive statistics will be applied to describe the participant's characteristics. The analyses will be adjusted for potential baseline confounders (at least age, sex, comorbidities, BMI and PA). Analysis will be performed using RStudio (R Foundation for Statistical Computing, Vienna, Austria) with statistical significance set at $\mathrm{p}<0.05$.

\section{Primary and secondary outcome measures}

Repeated measures analysis using linear mixed-effect models (LMMs) will be conducted to analyse the effect on clinical and structural outcomes. Variables will be described as mean with accompanying SD and categorical data as percentages. Time is categorised as baseline and follow-up for the analysis of mechanistic and structural outcomes. For self-reported outcomes on pain and activity limitations 3-monthly or 6-monthly intervals will be considered. An estimator of prediction error (Akaike information criterion, Bayesian information criterion) will be used to select the most appropriate model. The random-effect part of the LMM will include a subjectspecific intercept to account for within-subject correlations. The fixed-effect part of the LMMs consists of the predictors of interest: intervention, time and a treatment $\times$ time interaction. For each model, the estimated fixed effects with the accompanying $95 \% \mathrm{CI}$ and $\mathrm{p}$ value will be reported.
Logistic regression analysis will be conducted to analyse the effects on weight loss at 24 months. The change scores of body weight will be dichotomised into weight reduction $(<5 \%$ or $<5 \mathrm{~kg})$ and no weight reduction $(>5 \%$ or $>5 \mathrm{~kg}$ ). ORs and corresponding $95 \% \mathrm{CI}$ will be calculated.

\section{Cost-effectiveness analysis}

The cost-effectiveness and cost-utility analyses will be performed from a societal perspective. For the costutility analyses, the EQ-5D-5L health utilities will be used to calculate QALYs. Missing data will be imputed using Multiple Imputation by Chained Equations. ${ }^{44}$ The differences in mean QALYs and total societal costs between the intervention and control group will be assessed using a linear regression model. Incremental cost-effectiveness ratios (ICERs) will be calculated by dividing the difference in costs between the groups by the difference in effects. Statistical uncertainty surrounding costs and effects will be estimated using bias-corrected accelerated bootstrapping with 5000 replications and will be presented using cost-effectiveness planes to show the uncertainty surrounding the ICER. ${ }^{45}$ Cost-effectiveness acceptability curves will also be estimated showing the probability that the GLI is cost-effective compared with usual care alone for different ceiling ratios (ie, the amount of money that society is willing to pay for a unit of gained effect). ${ }^{46}$

\section{Supplementary analysis}

Causal mediation analysis will be conducted to estimate the effects of the GLI on clinical and structural outcomes at 24-month follow-up. The primary mediator, body weight and alternative mediators including PA and diet will be measured at 18 months. The analysis will be adjusted for possible confounders of the mediator-outcome effect. In addition, a per-protocol analysis will be performed according to attendance at the GLI sessions.

\section{Data monitoring and harms}

The GLI is an existing lifestyle programme that is recommended as part of standard care, hence, a data monitoring committee was not deemed to be necessary. Data monitoring will be carried out by an independent monitor once a year. Participants in the intervention group who experience adverse outcomes are instructed to discuss these with their GP and LSC. Serious adverse events will be reported to the Medical Research and Ethics Committee (METC).

\section{Patient and public involvement}

Patient representatives from the platform Artrose Gezond are involved at several stages of the trial. ${ }^{47}$ Patient representatives were involved in the development of the research question and the design of this trial. Patients will be actively involved in the recruitment of participants and dissemination of the final results by advising on appropriate strategies to reach our population and by providing comments on all participant information. For instance, a patient panel discussion was held to share ideas and offer opinions on how to optimise the recruitment. In 
addition, patient representatives will provide feedback on the topic guides that will be used during the semistructured interviews.

\section{ETHICS AND DISSEMINATION}

The trial received ethical approval from the METC of Erasmus MC University Medical Center Rotterdam (MEC 2020-0943). The protocol complies with the principles of Good Clinical Practice and the Declaration of Helsinki. Substantial modification must be approved by the METC before being implemented. All participants will provide written informed consent prior to participation in the trial. A unique numeric code will be assigned to every participant that bears no relation to personal data. Trial data will be stored in a locked place for a maximum of 15 years and will be available on reasonable request.

The results of the study will be disseminated in peerreviewed journals, national and international conferences and to participants, HCPs and the general population through presentations, websites and our patient platform.

\section{DISCUSSION}

This is the first RCT to evaluate the (cost-) effectiveness of the GLI in the treatment of patients with early-stage KOA. Given the health economic burden and the social impact of KOA for patients in daily living an effective lifestyle intervention must be identified that is tailored to the OA population.

Although prior research showed that weight loss is beneficial for improving pain and physical function in patients with established $\mathrm{KOA},{ }^{13-16}$ it remains unclear what the effect is in patients with early-stage KOA and whether this specific lifestyle intervention can lead to the $5 \%-10 \% \mathrm{wt}$ loss recommended in the guidelines. Also, there is still uncertainty whether weight loss can prevent or delay the structural progression of early-stage KOA. ${ }^{8}$ Besides, the potential working mechanisms by which the observed improvements in pain and physical function through a lifestyle intervention may be achieved are still not fully understood. In contrast to end-stage KOA, structural alterations in joint tissues at an earlier stage may be more susceptible to disease-modifying interventions, potentially slowing or preventing progressive change. Regardless of structural changes, patient-reported outcomes must be evaluated to better reflect clinically meaningful changes that are relevant to the target population and HCPs. Therefore, multiple primary outcomes are selected to fully capture the important effects of the GLI, in which a change in any primary outcome is clinically or scientifically meaningful, even in the absence of change in any other primary outcome.

With regard to the trial protocol, some strengths and limitations need to be addressed. First, the trial is pragmatic in design and will be performed in multiple centres enabling better generalisability and direct applicability to routine primary care settings. Additionally, the application of a mixed-methods approach will contribute to a potential successful implementation of the intervention. A possible limitation is that the GLI is individualised based on the participant's personal goals but also relies on the input of the healthcare professionals involved in the GLI. This may introduce clinical heterogeneity. Finally, an extended follow-up is required to assess long-term benefits after the end of the intervention.

A positive study outcome, thereby proving evidence of the effectiveness and cost-effectiveness of the GLI, is an important step to improve patient care and reduce the socioeconomic burden of KOA. A less positive outcome is indicative that the GLI in its present form is not suitable for patients with early-stage KOA. In either case, factors that may have influenced the outcome of the intervention will be evaluated in a qualitative study to further refine the intervention.

\section{Author affiliations}

${ }^{1}$ Department of General Practice, Erasmus Medical Center, Rotterdam, The Netherlands

2Department of Radiology \& Nuclear Medicine, Erasmus Medical Center, Rotterdam, The Netherlands

${ }^{3}$ Department of Health Sciences, Faculty of Science, Vrije Universiteit Amsterdam, Amsterdam, The Netherlands

${ }^{4}$ Dietician Practice in Primary Care, Diëtistenpraktijk HRC, Rotterdam, The Netherlands

${ }^{5}$ Research Center Innovations in Care, Rotterdam University of Applied Sciences, Rotterdam, The Netherlands

${ }^{6}$ Department of Internal Medicine, Erasmus Medical Center, Rotterdam, The Netherlands

${ }^{7}$ Department of Orthopaedics, Erasmus Medical Center, Rotterdam, The Netherlands

Contributors All authors (NEJJ, DS, E0, JB, JvT, AF, JR, JVM, MvM, SMAB-Z) contributed to the study conception and design. MvM obtained the funding and is responsible for the overall content as guarantor. NEJJ and MvM obtained ethical approval. Trial coordination, data collection, analysis and interpretation will be performed by NEJJ, MvM and SMAB-Z. The article was written by NEJJ and MvM. All other authors (DS, E0, JB, JvT, AF, JR, JVM, SMAB-Z) revised the manuscript and read and approved the submitted version.

Funding The work was supported by the Netherlands Organisation for Health Research and Development (ZonMW) (50-55515-98-004) and the Dutch Arthritis Society (ReumaNederland) (ZNW 20-501). The funders had no role in study design, data collection, analysis and interpretation of the data.

Competing interests None declared.

Patient consent for publication Not applicable.

Provenance and peer review Not commissioned; externally peer reviewed.

Supplemental material This content has been supplied by the author(s). It has not been vetted by BMJ Publishing Group Limited (BMJ) and may not have been peer-reviewed. Any opinions or recommendations discussed are solely those of the author(s) and are not endorsed by BMJ. BMJ disclaims all liability and responsibility arising from any reliance placed on the content. Where the content includes any translated material, BMJ does not warrant the accuracy and reliability of the translations (including but not limited to local regulations, clinical guidelines, terminology, drug names and drug dosages), and is not responsible for any error and/or omissions arising from translation and adaptation or otherwise.

Open access This is an open access article distributed in accordance with the Creative Commons Attribution Non Commercial (CC BY-NC 4.0) license, which permits others to distribute, remix, adapt, build upon this work non-commercially, and license their derivative works on different terms, provided the original work is properly cited, appropriate credit is given, any changes made indicated, and the use is non-commercial. See: http://creativecommons.org/licenses/by-nc/4.0/. 
ORCID iD

Nuria E J Jansen http://orcid.org/0000-0001-8804-9025

\section{REFERENCES}

1 NCD Risk Factor Collaboration (NCD-RisC). Worldwide trends in body-mass index, underweight, overweight, and obesity from 1975 to 2016: a pooled analysis of 2416 population-based measurement studies in 128.9 million children, adolescents, and adults. Lancet 2017;390:2627-42.

2 Fruh SM. Obesity: risk factors, complications, and strategies for sustainable long-term weight management. J Am Assoc Nurse Pract 2017;29:S3-14.

3 Hunter DJ, Bierma-Zeinstra S. Osteoarthritis. Lancet 2019;393:1745-59.

4 Rijksinstituut voor Volksgezondheid en Milieu (RIVM). Volksgezondheid Toekomst Verkenning 2018: RIVM, 2018. Available: https://www.vtv2018.nl/aandoeningen

5 Silverwood V, Blagojevic-Bucknall M, Jinks C, et al. Current evidence on risk factors for knee osteoarthritis in older adults: a systematic review and meta-analysis. Osteoarthritis Cartilage 2015;23:507-15.

6 Runhaar J, van Middelkoop M, Reijman M, et al. Malalignment: a possible target for prevention of incident knee osteoarthritis in overweight and obese women. Rheumatology 2014;53:1618-24.

7 Jiang L, Tian W, Wang Y, et al. Body mass index and susceptibility to knee osteoarthritis: a systematic review and meta-analysis. Joint Bone Spine 2012;79:291-7.

8 Landsmeer MLA, de Vos BC, van der Plas P, et al. Effect of weight change on progression of knee oa structural features assessed by MRI in overweight and obese women. Osteoarthritis Cartilage 2018;26:1666-74.

9 Nelson AE, Allen KD, Golightly YM, et al. A systematic review of recommendations and guidelines for the management of osteoarthritis: the chronic osteoarthritis management initiative of the U.S. bone and joint initiative. Semin Arthritis Rheum 2014;43:701-12

10 Ferket BS, Feldman Z, Zhou J, et al. Impact of total knee replacement practice: cost effectiveness analysis of data from the osteoarthritis initiative. BMJ 2017;356:j1131.

11 Hunter DJ, Schofield D, Callander E. The individual and socioeconomic impact of osteoarthritis. Nat Rev Rheumatol 2014:10:437-41.

12 Rijksinstituut voor Volksgezondheid en Milieu (RIVM). Kosten van Ziekten 2017: RIVM, 2017. Available: https://www.volksgezondheide nzorg.info/kosten-van-ziekten

13 Chu IJH, Lim AYT, Ng CLW. Effects of meaningful weight loss beyond symptomatic relief in adults with knee osteoarthritis and obesity: a systematic review and meta-analysis. Obes Rev 2018;19:1597-607.

14 Christensen P, Henriksen M, Bartels EM, et al. Long-Term weightloss maintenance in obese patients with knee osteoarthritis: a randomized trial. Am J Clin Nutr 2017;106:755-63.

15 Miller GD, Nicklas BJ, Davis C, et al. Intensive weight loss program improves physical function in older obese adults with knee osteoarthritis. Obesity 2006;14:1219-30.

16 Messier SP, Mihalko SL, Legault C, et al. Effects of intensive diet and exercise on knee joint loads, inflammation, and clinical outcomes among overweight and obese adults with knee osteoarthritis: the idea randomized clinical trial. JAMA 2013;310:1263-73.

17 Runhaar J, de Vos BC, van Middelkoop M, et al. Prevention of incident knee osteoarthritis by moderate weight loss in overweight and obese females. Arthritis Care Res 2016;68:1428-33.

18 Bierma-Zeinstra S, van Middelkoop M, Runhaar J, et al. Nonpharmacological and nonsurgical approaches in oa. Best Pract Res Clin Rheumatol 2020;34:101564.

19 Felson DT, Hodgson R. Identifying and treating preclinical and early osteoarthritis. Rheum Dis Clin North Am 2014;40:699-710.

20 Grässel S, Muschter D. Recent advances in the treatment of osteoarthritis. F1000Res 2020;9. doi:10.12688/ f1000research.22115.1. [Epub ahead of print: 0405 2020].

21 van Rinsum C, Gerards S, Rutten G, et al. The coaching on lifestyle (cool) intervention for overweight and obesity: a longitudinal study into participants' lifestyle changes. Int J Environ Res Public Health 2018;15. doi:10.3390/ijerph15040680. [Epub ahead of print: 0404 2018].

22 Pellegrini CA, Ledford G, Chang RW, et al. Understanding barriers and facilitators to healthy eating and physical activity from patients either before and after knee arthroplasty. Disabil Rehabil 2018;40:2004-10.

23 Marks R. Self-Efficacy and arthritis disability: an updated synthesis of the evidence base and its relevance to optimal patient care. Health Psychol Open 2014;1:2055102914564582.
24 Gunn AH, Schwartz TA, Arbeeva LS, et al. Fear of movement and associated factors among adults with symptomatic knee osteoarthritis. Arthritis Care Res 2017;69:1826-33.

25 Kanavaki AM, Rushton A, Efstathiou N, et al. Barriers and facilitators of physical activity in knee and hip osteoarthritis: a systematic review of qualitative evidence. BMJ Open 2017;7:e017042.

26 Chan A-W, Tetzlaff JM, Altman DG, et al. Spirit 2013 statement: defining standard protocol items for clinical trials. Ann Intern Med 2013;158:200-7.

27 Rijksinstituut voor Volksgezondheid en Milieu (RIVM). Interventieoverzicht Gecombineerde leefstijlinterventies, 2021. Available: https://interventies.loketgezondleven.nl/interventieoverz icht9/gli

28 Berendsen BA, Hendriks MR, Verhagen EA, et al. Effectiveness and cost-effectiveness of 'BeweegKuur', a combined lifestyle intervention in the Netherlands: rationale, design and methods of a randomized controlled trial. BMC Public Health 2011;11:815.

29 Greaves CJ, Middlebrooke A, O'Loughlin L, et al. Motivational interviewing for modifying diabetes risk: a randomised controlled trial. Br J Gen Pract 2008;58:535-40.

30 Miller WR. Motivational interviewing in service to health promotion. AJHP 2004;18:A1-10.

31 Nederlandse Diabetes Federatie (NDF). Voedingsrichtlijn voor diabetes 2006, 2006.

32 Health Council of the Netherlands. Guidelines for a healthy diet 2006, 2006.

33 Seidell JC, de Beer JJ, Kuijpers T. Richtlijn 'diagnostiek en behandeling van obesitas bij volwassenen en kinderen'. [Guideline 'Diagnosis and treatment of obesity in adults and children'. Ned Tijdschr Geneeskd 2008;152:2071-6.

34 Nederlands Huisartsen Genootschap (NHG). NHG-Richtljinen Niet Traumatische Knieklachten: Nederlands Huisartsen Genootschap (NHG), 2016. Available: https://www.nhg.org/standaarden/ samenvatting/niet-traumatische-knieklachten

35 Altman R, Asch E, Bloch D, et al. Development of criteria for the classification and reporting of osteoarthritis. classification of osteoarthritis of the knee. diagnostic and therapeutic criteria Committee of the American rheumatism association. Arthritis Rheum 1986;29:1039-49.

36 Maricar N, Callaghan MJ, Parkes MJ, et al. Interobserver and intraobserver reliability of clinical assessments in knee osteoarthritis. J Rheumatol 2016;43:2171-8.

37 Emery CA, Whittaker JL, Mahmoudian A, et al. Establishing outcome measures in early knee osteoarthritis. Nat Rev Rheumatol 2019;15:438-48.

38 Podsiadlo D, Richardson S. The timed "Up \& Go": a test of basic functional mobility for frail elderly persons. J Am Geriatr Soc 1991;39:142-8.

39 Assarsson E, Lundberg M, Holmquist G, et al. Homogenous 96plex pea immunoassay exhibiting high sensitivity, specificity, and excellent scalability. PLoS One 2014;9:e95192.

40 Boer CG, Radjabzadeh D, Medina-Gomez C, et al. Intestinal microbiome composition and its relation to joint pain and inflammation. Nat Commun 2019;10:4881.

41 Hunter DJ, Guermazi A, Lo GH, et al. Evolution of semi-quantitative whole joint assessment of knee oa: MOAKS (MRI osteoarthritis knee score). Osteoarthritis Cartilage 2011;19:990-1002.

42 Runhaar J, Schiphof D, van Meer B, et al. How to define subregional osteoarthritis progression using semi-quantitative MRI osteoarthritis knee score (MOAKS). Osteoarthritis Cartilage 2014;22:1533-6.

43 Wandel S, Jüni $\mathrm{P}$, Tendal B, et al. Effects of glucosamine, chondroitin, or placebo in patients with osteoarthritis of hip or knee: network meta-analysis. BMJ 2010;341:c4675.

44 Buuren Svan, Groothuis-Oudshoorn K. mice : Multivariate Imputation by Chained Equations in R. J Stat Softw 2011;45:67.

45 Thompson SG, Barber JA. How should cost data in pragmatic randomised trials be analysed? BMJ 2000;320:1197-200.

46 Fenwick E, O'Brien BJ, Briggs A. Cost-effectiveness acceptability curves--facts, fallacies and frequently asked questions. Health Econ 2004;13:405-15.

47 Erasmus MC Afdeling Huisartsgeneeskunde. Artrose Gezond: Artrose Gezond - Onderzoeksplatform voor mensen met Artrose, 2021. Available: https://artrosegezond.nl/

48 Wendel-Vos GCW, Schuit AJ, Saris WHM, et al. Reproducibility and relative validity of the short questionnaire to assess health-enhancing physical activity. J Clin Epidemiol 2003;56:1163-9.

49 Noyes FR, Mooar LA, Barber SD. The assessment of work-related activities and limitations in knee disorders. Am J Sports Med 1991;19:178-88.

50 Sangha O, Stucki G, Liang $\mathrm{MH}$, et al. The self-administered comorbidity questionnaire: a new method to assess comorbidity 
for clinical and health services research. Arthritis Rheum 2003:49:156-63.

51 Herr KA, Spratt K, Mobily PR, et al. Pain intensity assessment in older adults: use of experimental pain to compare psychometric properties and usability of selected pain scales with younger adults. Clin J Pain 2004;20:207-19.

52 van Laerhoven H, van der Zaag-Loonen HJ, Derkx BHF. A comparison of Likert scale and visual analogue scales as response options in children's questionnaires. Acta Paediatr 2004;93:830-5.

53 Janssen B, Szende A. Population norms for the EQ-5D, 2014: 19-30.

54 Janssen MF, Pickard AS, Golicki D, et al. Measurement properties of the EQ-5D-5L compared to the EQ-5D-3L across eight patient groups: a multi-country study. Qual Life Res 2013;22:1717-27.

55 Bouwmans C, Krol M, Severens H, et al. The iMTA productivity cost questionnaire: a standardized instrument for measuring and Valuing health-related productivity losses. Value Health 2015;18:753-8.

56 Roos EM, Toksvig-Larsen S. Knee injury and Osteoarthritis Outcome Score (KOOS) - validation and comparison to the WOMAC in total knee replacement. Health Qual Life Outcomes 2003;1:17.
57 Mehta SP, Sankar A, Venkataramanan V, et al. Cross-Cultural validation of the ICOAP and physical function short forms of the HOOS and KOOS in a multi-country study of patients with hip and knee osteoarthritis. Osteoarthritis Cartilage 2016;24:2077-81.

58 Hawker GA, Davis AM, French MR, et al. Development and preliminary psychometric testing of a new $\mathrm{OA}$ pain measure--an OARSI/OMERACT initiative. Osteoarthritis Cartilage 2008;16:409-14.

59 Jaeschke R, Singer J, Guyatt GH. Measurement of health status. ascertaining the minimal clinically important difference. Control Clin Trials 1989;10:407-15.

60 Conrozier T, Monet M, Lohse A, et al. Getting better or getting well? the patient acceptable symptom state (pass) better predicts patient's satisfaction than the decrease of pain, in knee osteoarthritis subjects treated with Viscosupplementation. Cartilage 2018;9:370-7.

61 Knapik A, Saulicz E, Gnat R. Kinesiophobia - introducing a new diagnostic tool. J Hum Kinet 2011;28:25-31.

62 Resnick B, Jenkins LS. Testing the reliability and validity of the selfefficacy for exercise scale. Nurs Res 2000;49:154-9. 